\title{
Los determinantes de la tasa de interés en El Salvador (1991 - 1999) Modelo de vectores autoregresivos
}

\section{Debates sobre la tasa de interés.}

En El Salvador, para abordar el estudio de la tasa de interés en la década que recientemente finalizó, hay que diferenciar dos etapas que corresponden a dos concepciones diferentes de la economía. Hasta abril de 1991 la política monetaria suponía la fijación de la tasa de interés por parte de la Junta Monetaria, mientras que a partir de esa fecha se opta por su liberación; es decir, se abandonan las políticas de intervención directa por parte de las autoridades monetarias y se enfatiza en las intervenciones indirectas, a través del desarrollo y utilización de mecanismos para el control de la oferta monetaria, como el encaje legal y las distintas modalidades y nominaciones que registran las operaciones de mercado abierto (OMAS).

A pesar de que este cambio ha permitido cierta reducción en la tasa de interés nominal, los resultados continúan siendo poco satisfactorios y el nivel de la tasa de interés sigue siendo percibida por la mayoría de sectores económicos como excesivamente elevado y, por tanto, limitado para la inversión y para el propio crecimiento económico.

Periódicamente, a través de los medios de comunicación se objeta a la autoridad monetaria por su incapacidad para corregir este problema y se recomiendan distintas medidas para abaratar el precio del dinero. En la mayoría de los casos, las críticas más fuertes se dirigen hacia las políticas de control monetario (OMAS y encaje legal) consideradas además por muchos sectores, como altamente restrictivas para la actividad económica. Por otra parte, tampoco el sistema bancario escapa a estas críticas, achacándosele ineficiencia, rigidez y prácticas oligopólicas.

Durante los primeros años de aplicación del nuevo modelo, las autoridades monetarias justificaban el mantenimiento de altas tasas de interés, con el argu- 
mento del diferencial de inflación con Estados Unidos, el cual ha venido perdiendo validez a medida que la inflación nacional ha llegado a los niveles internacionales'; ya que este fenómeno no ha estado acompañado de una reducción similar en la tasa de interés, que se mantiene muy por encima de los parámetros internacionales. Por tal razón, las quejas se dirigen ahora hacia la tasa de interés real (interés nominal menos inflación), ante lo cual las autoridades monetarias esgrimen la existencia de costos estructurales con repercusión en las tasas, como el riesgo cambiario ${ }^{2}$, el riesgo país, índice de morosidad, etc.

En cuanto a la banca, argumenta en su defensa que sus márgenes de intermediación no han variado sensiblemente en todos estos años, lo cual es relativamente cierto aunque puede indicar un reconocimiento explícito de su falta de eficiencia ${ }^{3}$, y responsabiliza también a las políticas de encaje y de OMAS de alto costo del dinero, además de repetir los argumentos de riesgo país, etc. En conversaciones sostenidas por estos autores con funcionarios bancarios, se llega a manifestar que "las tasas de interés pasivas (nominales) no se pueden reducir más, porque los depositantes no lo permitirían", es decir, se podría estar llegando a un piso estructural en cuanto a tasas de interés, ya sea por limitaciones internas del sistema financiero y/o por características estructurales de la economía salvadoreña, que impiden reducciones mayores en la tasa de interés.

La presión pública sobre este tema se ha agudizado en las fechas inmediatamente anteriores a la toma de posesión del nuevo gobierno del país. Los analistas económicos, las gremiales, los centros de estudios y demás generadores de opinión han criticado con dureza la situación y realizan propuestas de políticas alternativas, que permitirían en teoria, una reducción de las tasas de interés. Relacionado con ello, desde hace varios meses ha recobrado fuerza el debate entre estabilidad y crecimiento, que viene a poner en cuestión los resultados y la influencia de la política monetaria en la actividad económica.

Sin embargo, la mayoría de los cuestionamientos a las autoridades monetarias y al sistema bancario, así como las justificaciones ofrecidas por estas instancias, suelen descansar en análisis fragmentados que no captan el funcionamiento global del sistema económico. Los razonamientos que se plantean por una y otra parte, aunque retoman los principios de la teoría económica, parecieran partir más de ideas preconcebidas que del análisis de los hechos. La discusión ha llegado a tal punto, que recientemente se discutía en la Asamblea Legislativa la posibilidad de fijar de nuevo las tasas de interés por decreto, lo cual supondría una vuèlta a las políticas del pasado.

Independientemente de la razonabilidad en las posturas de los distintos sectores, lo cierto es que faltan análisis que clarifiquen el funcionamiento del sistema económico salvadoreño como conjunto y determinen en forma empírica, el impacto real de cada una de las variables sobre la tasa de interés, así como el efecto de ésta en el resto de variables macroeconómicas. En este sentido, se 
vuelve obligatorio la aplicación de técnicas econométricas para proporcionar contenido numérico a las afirmaciones teóricas sostenidas. Particularmente interesa comprobar si las políticas de control monetario aplicadas por el BCR contribuyen a incrementar la tasa de interés y su impacto en la actividad económica.

Por tanto, el presente análisis, fundamentado en un trabajo de tesis elaborado por los autores, pretende mostrar, utilizando el método de Vectores Autoregresivos (VAR), cuáles son las relaciones entre la tasa de interés y las principales variables macroeconómicas con que se relaciona, dentro de un sistema dinámico, en el que los cambios de una variable afectan al resto y cómo estas variaciones, en los siguientes períodos vuelven a afectar a todas aquellas de nuevo. Esto no resta validez a otros estudios y cálculos publicados, que por tener en muchos casos un carácter limitado, no permiten apreciar la dinámica completa del sistema.

Adicionalmente, para corroborar los resultados del VAR, se ha recurrido a la prueba de causalidad de Granger, que también permite inferir relaciones entre las variables consideradas.

\section{Determinación del modelo a utilizar}

El enfoque VAR consiste en la construcción y el análisis de un sistema de ecuaciones simultáneas, en el cual, todas las variables se consideran endógenas y son función lineal de los valores rezagados de esa misma variable y de todas las demás incluidas en el modelo; no obstante, en ocasiones cabe aceptar la incorporación de alguna variable exógena en el mismo, de acuerdo a la lógica y los criterios que rigen el estudio.

En este caso, los pasos que se han seguido para la construcción del modelo han sido los siguientes:

\subsection{Definición del periodo de estudio}

El periodo de estudio es establecido a partir del mes de abril de 1991 hasta marzo de 1999, utilizando una frecuencia mensual, con un total de 96 observaciones.

La frontera inferior del periodo a utilizar, obedece a que es a partir de esa fecha en que se da un cambio estructural en la economía salvadoreña, pasando de un sistema en buena medida planificado por el Estado, a una economía con mayor liberalización y desregulación, donde los precios son determinados en teoría por el espontáneo juego de la oferta y demanda. De manera similar, y por ser la tasa de interés el precio del dinero, después de estar determinada por el estado a lo largo de la década de los ochenta, pasa en la presente década, a estar determinada por múltiples factores cuya relación se intenta descubrir.

La última fecha considerada en este trabajo, la limitó la disponibilidad de datos actualizados, obtenidos en su mayoría en las estadísticas del Banco Central de Reserva de El Salvador, de cuyos funcionarios se ha obtenido una excelente colaboración. 


\subsection{Elección y definición de las variables a utilizar}

Pese a que los modelos VAR se consideran a-teóricos y sólo pretenden descubrir las relaciones entre dos o más series de tiempo, se ha procedido a la selección de las variables que de acuerdo a la teoría económica tienen importantes relaciones con la tasa de interés.

En el Cuadro № 1 se presenta un resumen de las variables consideradas y de la variable "proxy" utilizada para su medición.

\subsection{Transformaciones realizadas a las variables}

A fin de incluir a las variables antes mencionadas en un solo modelo, se han realizado varias operaciones a los datos iniciales. Concretamente, las series de los índices de precios al consumidor, como proxy de la inflación tanto nacional como internacional (IPCN e IPCUSA), debieron convertirse al mismo año base para que su análisis fuese comparable. Se optó por utilizar la base de enero/90.

En general, además de la diferenciación realizada durante las pruebas de raíz unitaria, las series sufrieron dos transformaciones principales antes de elaborar con ellas algún modelo VAR. La decisión de cual de las transformaciones utilizar, dependió únicamente de la naturaleza de la serie.

\section{Cuadro № 1"}

Variables a considerar en el modelo

\begin{tabular}{|c|c|c|}
\hline Endogenas & Proxy & Abreviatura \\
\hline Tasa de interés nacional & Tasas de Intcrés Activas` Promedio Ponderado del año & INAC \\
\hline Tasa de inflación nacional & $\begin{array}{l}\text { Indice de Precios al Consumidor', basc }=100 \text { en dicicm- } \\
\text { bre de } 1992 .\end{array}$ & IPCN \\
\hline Producto Nacional & $\begin{array}{l}\text { Indice de Volumen de la Actividad Económica, año } \\
\text { base }=100 \text { en 1990. }\end{array}$ & IVAE \\
\hline \multirow[t]{2}{*}{ Olerta Monclaria ${ }^{7}$} & $\begin{array}{l}\text { Agregado monctario M2, compuesto por el medio circu- } \\
\text { lantc, depósilos de ahorro y depósilos a plazo. }\end{array}$ & M2 \\
\hline & $\begin{array}{l}\text { Agregado monclario } \mathrm{M} 3 \text {, compuesto por M2 más depó- } \\
\text { silos cn moneda extranjera y lítulos valores. }\end{array}$ & M3 \\
\hline Politica Monetaria $^{\mathrm{K}}$ & $\begin{array}{l}\text { Coeficiente de esterilización de depósitos bancarios. } \\
\text { Calculado como el cociente del saldo de CAM y CEM } \\
\text { mantenidos por los bancos y financieras, entre el total } \\
\text { de depósitos registrados en esas instiluciones. } \\
\text { Cocficiente de csterilización de depósitos bancarios, in- } \\
\text { cluyendo la tasa de cncuje lcgal. Calculado como cl co- } \\
\text { cicnic del saldo de CAM y CEM mantcnidos por los } \\
\text { bancos y financicras, más las rescrvas cxigidas por } \\
\text { cl cncajc lcgal, dividido entre cl lolal de depósitos } \\
\text { regisılrados cn csas instiluciones. }\end{array}$ & TVPASENC \\
\hline
\end{tabular}




\begin{tabular}{l|l|c}
\hline \multicolumn{1}{c|}{ Endogenas } & \multicolumn{1}{c|}{ Proxy } & Abreviatura \\
\hline Tasa de interés internacional & $\begin{array}{l}\text { Tasa de interés de los bonos del lesoro de los EEUU, } \\
\text { a } 180 \text { dias plazo. }\end{array}$ & BONOS \\
\hline $\begin{array}{l}\text { Tasa de inflación } \\
\text { internacional }\end{array}$ & $\begin{array}{l}\text { Indice de precios al cónsumidor urbano de los EEUU } \\
\text { Base }=100 \text { entre los años } 1982-1984\end{array}$ & IPCUSA \\
\hline Tasa de encaje legal & $\begin{array}{l}\text { Promedio ponderado de las lasas de encaje legal apli- } \\
\text { cadas a los depósilos más significalivos }\end{array}$ & ENCAJE \\
\hline
\end{tabular}

Los tipos de transformación practicadas, fueron:

- Transformación en diferencia estacionaria. Se practicó sobre aquellas series que ya presentaban los datos en forma de porcentaje; tal es el caso de la tasa de interés nacional e intemacional (INAC y BONOS); las series que representaban coeficientes (TVPAS y TVPASENC); y finalmente el promedio ponderado del encaje (ENCAJE).

- Transformación en diferencia logarítmica estacionaria. Se practicó sobre las restantes series de variables, ya que al hacer uso de logaritmos se obtienen las tasas de crecimiento de las mismas, efecto que ya tenían las demás series al encontrarse en forma de porcentaje. Las series afectadas por este cambio son las correspondientes a los agregados monetarios (M2 y M3); las que representan índices de precios al consumidor (IPC e IPCUSA); y finalmente el índice de la actividad económica (IVAE).

Nótese que en ambos casos las transformaciones han incluido el término estacionario a doce meses; esto se realizó luego de observar que muchas de las series económicas presentan tendencias repetitivas año con año; de aquí que se busque suavizar su evolución en el tiempo al incluir el componente estacional, para obtener lo que estadísticamente se conoce como series estacionarias.

\subsection{Elaboración de Diferentes Modelos VAR}

En cuanto a las variables a incluir en el modelo, una vez realizadas las transformaciones descritas anteriormente, se procedió a diseñar diferentes pre-modelos (ccmbinaciones de variables) a fin de determinar cuales de las variables proxy proporcionan mayor representatividad.

Todos los pre-modelos se analizaron con $12,9,6,3,2$ y 1 rezagos, aplicando distintas pruebas para tres diferentes grupos de combinaciones, con el fin de obtener gradualmente el que mejor explica la realidad salvadoreña; en todos ellos se consideran como variables principales, INAC, IPCN e IVAE. Junto a ellas, se fueron incluyendo en forma paulatina y analizando la oferta monetaria, la esterilización y las variables exógenas, con objeto de obtener un modelo representativo.

\subsection{Estimación de Rezagos}

Se debe actuar con cautela, no sólo en la selección de las variables a introducir, sino también en la identificación de la cantidad óptima de rezagos que per- 
mita trabajar con un modelo significativo o confiable. La teoría econométrica sostiene que idealmente se debe utilizar un número grande de rezagos, para permitir capturar en el modelo el tiempo necesario para que una variable afecte a otra. Sin embargo, un mayor número de coeficientes a estimar, ocasiona problemas de sobreparametrización (o ausencia de parsimonia) y casi siempre incrementa las posibilidades de multicolinealidad entre las ecuaciones, lo que lleva a estimaciones ineficientes de los valores de los coeficientes y a graves errores de pronóstico al utilizar el modelo en valores fuera de la muestra.

Otro inconveniente al usar un número grande de rezagos es que consume muchos grados de libertad, lo que restringe el número de variables que se pueden incluir en el sistema; la introducción de variables adicioṇales requiere preferiblemente que se incremente el tamaño de la muestra.

Con el fin de determinar el número apropiado de rezagos, existen diversas pruebas y estadísticos, los cuales no necesariamente conducen a los mismos resultados, sin embargo, permiten una mejor aproximación a las variables y al número óptimo de rezagos que debe poseer el modelo que se intenta establecer. En el presente trabajo se han utilizado inicialmente los estadisticos Akaike ${ }^{12} \mathrm{Y}$ Schwarz ${ }^{13}$; y después las pruebas $L^{14}$ y Asintótica ${ }^{15}$, para evaluar el tamaño del rezago que mejor resultado proporciona en los modelos evaluados.

\subsection{Análisis del Estadístico "t". Nivel de Significancia de los Coeficientẹ Estimados.}

En los pre-modelos que mejores resultados presentaban en las pruebas LR y Enders, se aplicó la prueba del " $t$ " estadístico, estimado para cada coeficiente, evaluándose si estos son significativos o no, para precisar el número de rezagos que mejores resultados ofrece.

\section{Función Impulso Respuesta}

Con el objetivo de capturar las relaciones dinámicas existentes entre las variables del modelo VAR y por la dificultad de interpretar los coeficientes estimados en él, se utiliza como herramienta la Función Impulso Respuesta (IRF), que permite obtener algunas conclusiones sobre las relaciones entre variables.

La función impulso respuesta muestra cómo reacciona una variable endógena ante una innovación positiva, y cómo, debido al carácter dinámico del sistema, responden las demás variables endógenas. Por tanto, un aspecto muy importante a considerar en el momento de realizar la función impulso respuesta es el ordenamiento que se les dará a las variables ${ }^{1 \mathrm{~h}}$, especialmente en modelos con errores correlacionados.

En el modelo propuesto, se presentan en general bajos valores de correlación entre las variables, lo cual es posible verificar aplicando el siguiente criterio: 
Cuadro No. 2

Coeficientes del modelo

Muestra (ajustada): $\quad$ 1992:07. 1999:03

Observaciones incluidas: $\quad 81$

Errores Estándar y “t” Estadísticos

\begin{tabular}{l|c|c|c|c|c}
\hline & DE_INAC & DE-IPCN & DE_IVAE & DE_M3 & DE_TVPAS \\
\hline DE_INAC (-1) & $\mathbf{0 . 3 9 4 8}$ & -0.1791 & 0.9448 & 0.1778 & 0.3658 \\
& $\mathbf{- 3 . 3 5 8 6}$ & -0.6828 & 1.4581 & $\mathbf{0 . 4 2 8 7}$ & 1.0516 \\
DE_INAC (-2) & $\mathbf{0 . 1 9 9 8}$ & 0.3438 & $\mathbf{- 2 . 6 8 4 9}$ & -0.5883 & -0.1259 \\
& $\mathbf{1 . 6 7 2 1}$ & 1.2852 & $\mathbf{- 4 . 0 6 3 6}$ & -1.3908 & -0.3551 \\
DE_IPCN (-1) & 0.0125 & $\mathbf{0 . 2 7 7 5}$ & -0.3434 & $\mathbf{0 . 3 3 0 6}$ & 0.1585 \\
& 0.2273 & $\mathbf{2 . 2 6 0 9}$ & -1.1325 & $\mathbf{1 . 7 0 3 3}$ & 0.9739 \\
E_IPCN (-2) & $\mathbf{0 . 0 9 7 9}$ & 0.1570 & -0.2264 & -0.3276 & -0.2803 \\
& $\mathbf{1 . 7 0 2 9}$ & 1.2237 & -0.7143 & -1.6141 & -1.6472 \\
DE_IVAE (-1) & $\mathbf{- 0 . 0 3 9 3}$ & $\mathbf{0 . 0 1 7 2}$ & $\mathbf{- 0 . 6 2 5 6}$ & -0.0358 & -0.0111 \\
& $\mathbf{- 2 . 0 5 1 2}$ & $\mathbf{0 . 4 0 3 0}$ & $\mathbf{- 5 . 9 2 5 0}$ & -0.5291 & -0.1965 \\
DE_IVAE (-2) & -0.0132 & 0.0310 & $\mathbf{- 0 . 2 5 4 3}$ & 0.0153 & -0.0366 \\
& -0.6954 & 0.7298 & $\mathbf{- 2 . 4 2 6 1}$ & 0.2277 & -0.6509 \\
DE_M3 (-1) & 0.0497 & 0.0311 & $\mathbf{- 0 . 4 7 5 5}$ & $\mathbf{- 0 . 3 2 3 5}$ & -0.0987 \\
& 1.3455 & 0.3768 & $\mathbf{- 2 . 3 3 4 5}$ & $\mathbf{- 2 . 4 8 0 7}$ & -0.9025 \\
DE_M3 (-2) & 0.0124 & -0.1059 & -0.1103 & -0.0428 & -0.1005 \\
& 0.3243 & -1.2388 & -0.5223 & -0.3169 & -0.8872 \\
DE_TVPAS (-1) & -0.0702 & -0.1460 & 0.3871 & $\mathbf{0 . 3 4 0 0}$ & $\mathbf{0 . 2 6 6 0}$ \\
& -1.1518 & -1.4148 & 1.5192 & $\mathbf{2 . 0 8 4 0}$ & $\mathbf{1 . 9 4 4 8}$ \\
DE_TVPAS (-2) & -0.0345 & -0.1113 & 0.2852 & -0.1813 & 0.0363 \\
& -0.7163 & -1.0350 & 1.0735 & -1.0659 & 0.2548 \\
C & 0.0001 & -0.0009 & -0.0023 & -0.0024 & -0.0013 \\
& 0.2405 & -0.6809 & -0.7131 & -1.1376 & -0.7291 \\
DE_ENCAJE & 0.1654 & 0.2917 & $\mathbf{- 1 . 7 8 9 5}$ & -0.2063 & 0.2166 \\
& 1.1995 & 0.9481 & $\mathbf{- 2 . 3 5 4 6}$ & -0.4240 & 0.5308 \\
DE_BONOS & -0.1800 & -0.3671 & -0.2070 & 0.6442 & -0.1994 \\
& -0.8170 & -0.7469 & -0.1705 & $\mathbf{0 . 8 2 8 9}$ & -0.3061 \\
DE_IPCUSA & -0.2651 & -0.7805 & 1.2954 & -0.9375 & 0.1142 \\
& -0.5858 & -0.7730 & $\mathbf{0 . 5 1 9 3}$ & -0.5871 & 0.0853 \\
\hline
\end{tabular}

En negrita se reseñan los coefícientes en los que el " $t$ " estadístico es mayor al " $t$ " crítico.

Para un nivel de significancia del 5\% en la curva de Student, el " $t$ " crítico es 1.671 
$\mathbf{H}_{\mathbf{0}}$ : Hipótesis Nula:

$\mathrm{H}_{1}$ : Hipótesis Alternativa:
Coeficiente de Correlación estimado $<0.20$ "No existe correlación entre ambas variables"

Coeficiente de Correlación estimado $>0.20$ "Existe correlación entre ambas variables"

\section{Cuadro № 3}

Matriz de correlaciones

\begin{tabular}{l|r|r|r|r|r}
\hline & DE_INAC & DE_IPCN & DE_TVPAS & DE_M3 & DE_IVAE \\
\hline DE_INAC & $\mathbf{1 . 0 0 0 0 0 0 0}$ & -0.0748990 & 0.0840257 & 0.0070813 & $\mathbf{0 . 3 1 7 7 7 9}$ \\
DE_IPCN & -0.0748990 & 1.0000000 & -0.297054 & 0.0350119 & -0.1893715 \\
DE_TVPAS & 0.0840257 & -0.297054 & $\mathbf{1 . 0 0 0 0 0 0 0}$ & $\mathbf{0 . 3 8 0 7 0 5}$ & $\mathbf{0 . 2 2 5 3 3 3 0}$ \\
DE_M3 & 0.0070813 & 0.0350119 & $\mathbf{0 . 3 8 0 7 0 5}$ & $\mathbf{1 . 0 0 0 0 0 0 0}$ & -0.1122731 \\
DE_IVAE & $\mathbf{0 . 3 1 7 7 7 9}$ & -0.1893715 & $\mathbf{0 . 2 2 5 3 3 3 0}$ & -0.1122731 & $\mathbf{1 . 0 0 0 0 0 0 0}$ \\
\hline
\end{tabular}

Según el Cuadro No. 3, los pares de variables que presentan algún nivel de correlación son los relativos a TVPAS: TVPAS-IPCN, TVPAS-M3 y TVPASIVAE, además de INAC-IVAE, aunque los valores estimados no son excesivamente altos.

Teniendo presente esto, se realizaron varias pruebas de impulso respuesta utilizando distintos ordenamientos, siempre manteniendo INAC en primer lugar; esto permite determinar que los efectos de las innovaciones de INAC en otras variables, $y$ las respuestas de INAC ante innovaciones de otras variables, son similares para todos los ordenamientos, donde INAC figure en primer lugar.

Aunque cabe señalar que al variar el ordenamiento, se presentan variaciones a corto plazo cuando se trata de las funciones de impulso respuesta que relacionan a algunas de las otras variables distintas al INAC Estas diferencias sólo afectan a las respuestas del primer mes, no a los multiplicadores de largo plazo, por lo que el dilema se presenta sobre el carácter contemporáneo o no contemporáneo de las respuestas de determinadas variables ante impulsos positivos en otras, aunque sin afectar las conclusiones que se ofrecen en el presente trabajo.

Por tanto, ya que el ordenamiento no afecta a INAC, se ha optado analizar la función impulso respuesta bajo un solo ordenamiento, INAC, M3, TVPAS, IPCN,

- IVAE, el cual se justifica por las siguientes razones:

- INAC, por la incidencia evidente sobre sí misma

- M3 y TVPAS, por ser variables monetarias con supuesta incidencia inmediata con INAC. 
- IPCN e IVAE, por ser variables cuyo ajuste con INAC se produce en forma más lenta.

Por otra parte, cabe señalar que el análisis de impulso respuesta se ha realizado en las dos direcciones; en primer lugar, comprobando la respuesta de INAC ante innovaciones de sí misma y de las demás variables endógenas; y en segundo lugar, revisando las respuestas de las variables endógenas del modelo ante innovaciones en INAC. Además, para corroborar algunos resultados se han revisado relaciones entre variables distintas de INAC, así como las respuestas en otros ordenamientos, que vienen a reforzar las conclusiones extraídas.

Por otra parte para corroborar las relaciones entre variables, también se recurre a la prueba de causalidad de Granger, la cual intenta distinguir si existe alguna relación estadística entre dos series de tiempo, distinguiendo la dirección de la causalidad según sea el caso. ${ }^{17}$

Si bien no existe un parámetro preciso que permita determinar cuando existe causalidad Granger o no, en un trabajo anterior presentado por Hurtado y Osorio" ${ }^{1 \mathrm{k}}$, se adopta el criterio de 0.20 y valores menores para rechazar la hipótesis nula $\left(\mathrm{H}_{11}\right)$.

De aquí que se defina:

$\mathrm{H}_{t}:$ : Hipótesis Nula. "Variable A no causa Granger a la Variable B"

$\mathrm{H}_{1}$ : Hipótesis Alternativa "Variable A causa Granger a la Variable B"

En la medida en que sea oportuno, en los siguientes apartados se profundizará sobre las relaciones Granger presentes entre las variables del modelo.

Pero regresando al enfoque VAR, la IRF permite analizar la respuesta de una variable ante innovaciones positivas de otra, en el largo y en el corto plazo.

\subsection{El Impulso Acumulado}

Si se desea conocer el efecto acumulado de los impulsos de cada variable endógena sobre la tasa de interés, se puede optar por sumar los multiplicadores ó el valor numérico que la función impulso respuesta toma en los diferentes meses, lo cual nos indica el sentido y la magnitud del impulso acumulado al mes que se desee.

La misma operación se puede hacer en sentido contrario, para determinar el impacto acumulado 'de la innovación de la tasa de interés sobre las demás variables endógenas. Cabe añadir que la función de impulso respuesta se aplica sobre variables transformadas, por tanto, no se puede inferir los valores que adquieren las variables, aunque sí el sentido de la respuesta, expresada en porcentajes. 


\subsubsection{Innovaciones de las variables endógenas sobre INAC:}

Como se aprecia en el Cuadro № 4, el sentido de la respuesta de la tasa de interés ante innovaciones positivas de cada variable endógena se mantiene con el mismo signo para todos los períodos. Por tanto, cabe afirmar que a largo plazo, INAC responde en forma positiva ante innovaciones positivas de ella misma, de la cantidad de dinero nominal y de la inflación; y ofrece respuestas negativas ante innovaciones positivas en la esterilización y en la actividad económica.

\section{Cuadro № 4}

Impacto acumulado de las innovaciones de las variables endógenas sobre INAC

\begin{tabular}{l|c|c|c|c|c}
\hline & DE_INAC & DE_M3 & DE_TVPAS & DE_IPCN & DE_IVAE \\
\hline A 12 meses & 0.0094812681 & 0.0002536771 & -0.0092513271 & 0.0058045993 & -0.0008935241 \\
A 15 meses & 0.0094976813 & 0.0001884498 & -0.0095532594 & 0.0060260054 & -0.0008673818 \\
A 24 meses & 0.0094979895 & 0.0001532582 & -0.0097039078 & 0.0061378541 & -0.0008523672 \\
A 36 meses & 0.0094976721 & 0.0001519617 & -0.0097087544 & 0.0061415461 & -0.0008517935 \\
A 48 meses & 0.0094976675 & 0.0001519518 & -0.0097087870 & 0.0061415715 & -0.0008517890 \\
A 60 meses & 0.0094976675 & 0.0001519517 & -0.0097087871 & 0.0061415716 & -0.0008517889 \\
\hline
\end{tabular}

Otro elemento a destacar es el orden de las variables en cuanto a su impacto sobre INAC. Para 12 meses, el orden es INAC, TVPAS, IPCN, IVAE y M3. Pero a partir del $15^{\circ}$ mes, es TVPAS la de mayor impacto, mientras que la propia INAC queda en segundo lugar, manteniéndose el resto sin cambios.

Estos resultados muestran que el comportamiento a largo plazo de INAC, ante cambios en INAC, IPCN e IVAE es consecuente con la teoría económica (en el caso de IVAE con algunas reservas), mientras que la respuesta ante M3 y TVPAS es contraria a la lógica económica elemental. Como se trata de demostrar más adelante, la causa de esto es que las innovaciones de M3 y TVPAS, a través del estímulo que ejercen sobre otras variables, vienen a causar en INAC un efecto contrario a lo esperado por la teoría económica.

\subsubsection{Innovaciones de INAC sobre las variables endógenas.}

En el Cuadro № 5 , el sentido de la respuesta de las variables endógenas ante innovaciones positivas en la tasa de interés también se mantiene con el mismo signo. Por tanto, cabe afirmar que a largo plazo, las variables endógenas que responden en forma positiva ante innovaciones positivas de INAC, son ella misma y el TVPAS, mientras que la cantidad de dinero, la inflación y la actividad económica responden en forma negativa. 


\section{Cuadro № 5}

Impacto acumulado de las innovaciones de INAC sobre el resto de variables endógenas

\begin{tabular}{l|c|c|c|c|c}
\hline & DE_INAC & DE_M3 & DE_TVPAS & DE_IPCN & DE_IVAE \\
\hline A 12 meses & 0.0094812681 & -0.0020534200 & 0.0057601733 & -0.0010818074 & -0.0014161894 \\
A 15 meses & 0.0094976813 & -0.0020773851 & 0.0057890229 & -0.0010825207 & -0.0014347758 \\
A 24 meses & 0.0094979895 & -0.0020770426 & 0.0057955615 & -0.0010866454 & -0.0014284773 \\
A 36 meses & 0.0094976721 & -0.0020769005 & 0.0057953855 & -0.0010868172 & -0.0014280816 \\
A 48 meses & 0.0094976675 & -0.0020768977 & 0.0057953816 & -0.0010868190 & -0.0014280758 \\
A 60 meses & $\mathbf{0 . 0 0 9 4 9 7 6 6 7 5}$ & -0.0020768977 & 0.0057953816 & -0.0010868190 & -0.0014280757 \\
\hline
\end{tabular}

En cuanto al orden de las variables por su respuesta a la innovación de INAC, para 12 meses el orden es INAC, TVPAS, M3, IVAE e IPCN, manteniéndose sin cambios el resto de períodos.

Con ello se confirma la importancia de la relación entre INAC y TVPAS. Estos resultados indican que el comportamiento de todas las variables puede ser consecuente con la teoría económica, excepto en el caso del IPCN. En el siguiente apartado se volverá sobre las implicaciones económicas que suponen estos resultados.

\subsection{Procesos de ajuste que siguen las respuestas.}

Tan interesante como determinar las relaciones a largo plazo entre dos variables, es determinar sus respuestas en corto plazo y el ajuste que se sigue hasta su estabilización en el tiempo.

A continuación se presentan las relaciones de impulso respuesta entre INAC y cada una de las variables endógenas, tanto en sentido de la respuesta de INAC ante innovaciones en cada variable, como la respuesta de cada variable ante innovaciones de INAC. Para facilitar la comprensión de algunos razonamientos que a continuación se exponen, resulta imprescindible analizar las gráficas relativas a las distintas IRFs del modelo.

\subsubsection{INAC sobre sí mismo:}

En el período estudiado, la función de impulso respuesta indica que las variaciones positivas de la tasa de interés tienen un efecto positivo contemporáneo sobre esa misma varíable, tal como se aprecia en la Gráfica № 1 . Es decir, su efecto máximo sobre la propia INAC se produce en el primer mes, perdurando dicho efecto en forma sensible casi por un año, en que se estabiliza de nuevo la variable, lo cual es una muestra de su inercia. Aunque entre las variables del modelo, es la que menos impacto tiene sobre sí misma; en cambio, IVAE y M3, con variaciones más acusadas, son las que más impacto tienen sobre sí mismas. 


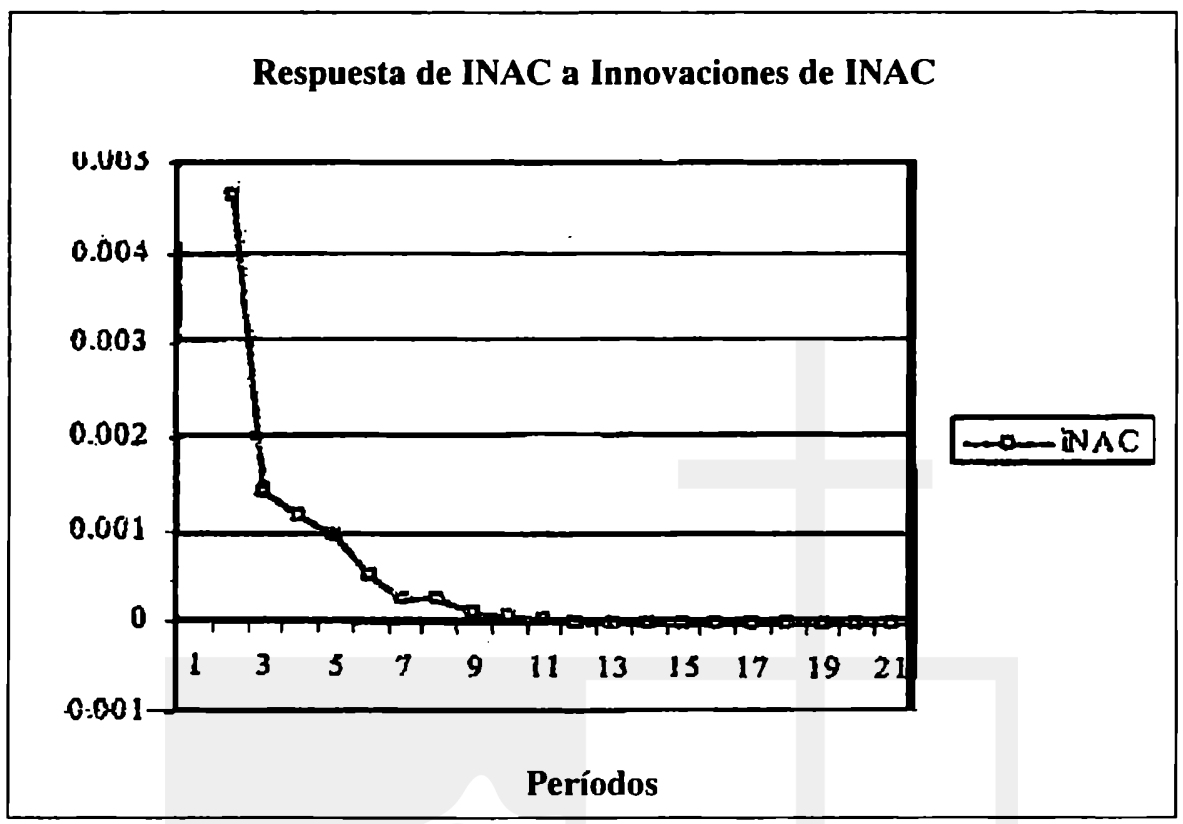

\subsubsection{M3 sobre INAC}

La función de impulso respuesta que analiza los cambios en la tasa de interés, producidos por variaciones positivas de la cantidad de dinero (ver Gráfica No. 2), indica un efecto positivo sobre la tasa de interés, no contemporáneo, alcanzando su mayor impacto en el segundo mes y continuando sus efectos en forma sensible hasta el $6^{0}$ mes; a partir de ahí se diluye el impacto, aunque su efecto acumulado es positivo.

Esta situación contraria en alguna medida a la teoría económica, que sostiene que un incremento en la cantidad de dinero hace disminuir la tasa de interés.

La interpretación de este comportamiento, es que una innovación positiva en M3, implica una respuesta contemporánea positiva en TVPAS, a lo que hay que añadir una ligera respuesta positiva de IPCN también en el primer mes, las cuales contrarrestan la expansión monetaria inicial.

En el segundo mes este efecto contractivo es recogido por el incremento de INAC, que incluso es acompañado por una caída espectacular de M3 a consecuencia de la inercia que presenta esta variable.

Por el contrario, en el tercer mes la caída de TVPAS y de IPCN, junto a la recuperación de $M 3$, suponen una expansión de la oferta monetaria real y por tanto, a partir de ese período comienza a caer la respuesta de la tasa de interés. 
Por tanto, ante una innovación positiva de M3, nos encontramos con 2 meses inicjales de con-tracción de la oferta monetaria real, por la política de OMAS y la inercia de la propia M3, que se revierte a partir del tercer mes.

Coincidentemente, ante una innovación de M3, el IVAE se reduce en los 2 primeros meses, para incrementarse a partir del tercer, cuando empieza la expansión de la oferta monetaria real.

Las relaciones de M3 e INAC no quedan reflejadas claramente en la prueba de causalidad de Granger (ver Anexo 1).

\subsection{TVPAS sobre INAC}

La función de impulso respuesta que analiza los cambios en la tasa de interés ante la política de regulación de la liquidez a corto plazo (ver Gráfica No. 3), indica que innovaciones positivas en las OMAS producen una respuesta negativa no contemporánea en la tasa de interés, cuyo efecto se extiende hasta aproximadamente el $14^{\circ}$ mes.

Esta fuerte relación que vienc avalada por la prueba Granger (ver Anexo 1), implica un resultado sorprendente, ya que se esperaria que los impulsos positivos en las políticas de restricción monetaria (restricción de la liquidez), contribuyan a incrementar la tasa de interćs. Por tanto, cste fenómeno requeriria de un estudio más profundo y detenido sobre el tema.

En el modelo aplicado y el ordenamiento seguido, la innovación positiva de TVPAS no produce una respuesta contemporánea en INAC, pero sí tiene impacto negativo en los precios desde el primer mes, por lo que se contrantesta el efecto restrictivo de TVPAS en lo referente a la oferta monetaria real.

En cl segundo mes, cuando se recogen los efeclos de los cambios en todas las variables los precios continúan ofreciendo una respuesta negaliva, pcro adcmás sc incrementa $\mathrm{M} 3$, cs decir, sc prolonga cl cfecto de expansión de la ofcrta monctaria rcal y, por tanto, siguc caycndo INAC.

En el tercer mes la innovación positiva inicial de TVPAS va a tener su máximo impacto negativo sobre la tasa de interés debido a que se reduce M3, pero TVPAS registra valores próximos a 0 e IPCN sigue ofreciendo respuestas negativas aunque crecicntcs. (iráfica No 2

Respuesta a Innwaciones de MB

INAC

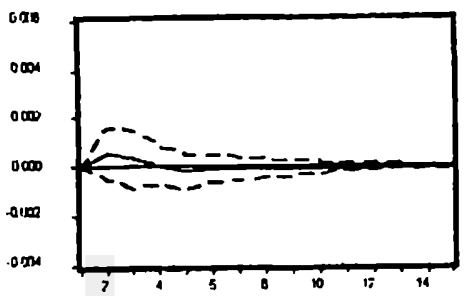

M3

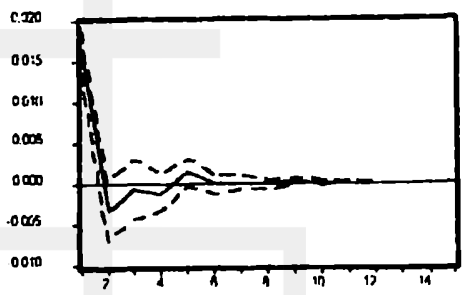

TVPAS
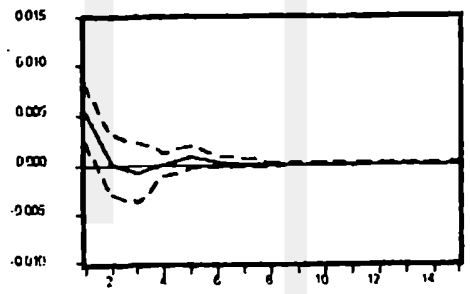

IPCN

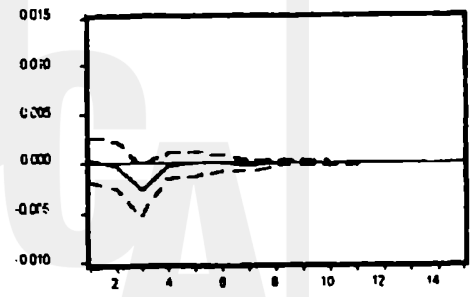

NAE

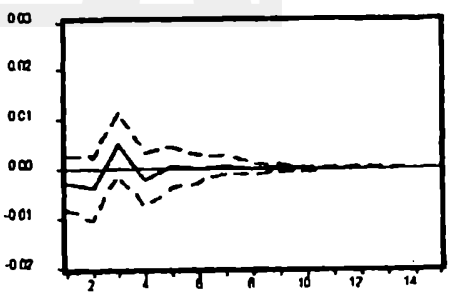


También en este caso existe coincidencia en cuanto a la respuesta de IVAE, que por la expansión de la oferta monetaria real y el consiguiente descenso de INAC, registra respuestas positivas aunque decrecientes durante los tres primeros meses.

Posteriormente a partir del $4^{\circ}$ mes, cambia la tenden- .mo cia en la respuesta de INAC ante la innovación positiva oom inicial de TVPAS; la respuesta de la tasa de interés em- ove pieza a crecer, ya que hay un aumento en $\mathrm{M} 3$ y en IVAE y se va diluyendo la respuesta negativa de IPCN. A partir de ese mes, las variables alcanzan la estabilidad, excepto INAC, que por la fuerza de TVPAS, tarda más tiempo en equilibrarse.

De acuerdo con esta interpretación, la política de OMAS crea una expansión de la oferta monetaria real, ...1s debido a que se muestra sumamente eficaz en el control de la inflación y por tanto, aunque parczca paradójico, de acuerdo al modelo aplicado contribuye a contener la tasa de interés nominal. De hecho como se menciona con anterioridad, a pesar de la política de esterilización aplicada, la tasa de interés nominal de El Salvador ha venido cayendo en el período analizado y cs sensiblemente inferior a la de otros países del mismo entorno, aunque esta reducción no sca suficiente.

\subsubsection{IPCN sobre INAC:}

La función de impulso respuesta que analiza los cambios en la tasa de interés producidos por variaciones positivas de la inflación (ver Gráfica $\mathrm{N}^{2} 4$ ), indica un efecto positivo no contemporáneo de los precios sobre la tasa de interés, que alcanza su mayor impacto en el tercer, aunque su influencia sensible se extiende casi por un año. Su efecto acumulado es positivo.

Existe consistencia con los resultados del análisis Granger, en donde la probabilidad de rechazar $\mathrm{H}_{0}$ es muy baja, presentándose, por tanto, allas probabilidades de aceptar la causalidad de IPCN sobre INAC (ver Anexo 1).
Grifica Na J

Respoeata a Innovaciones de TVPAS

INAC

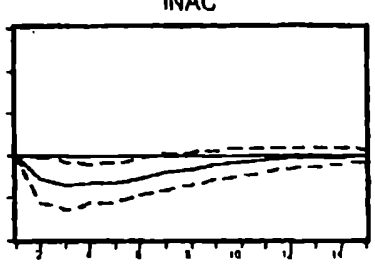

M3

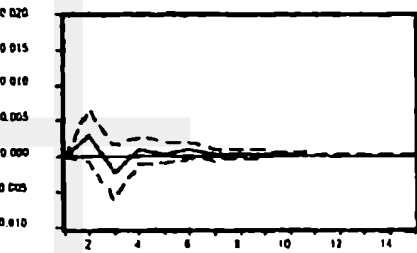

TVPAS

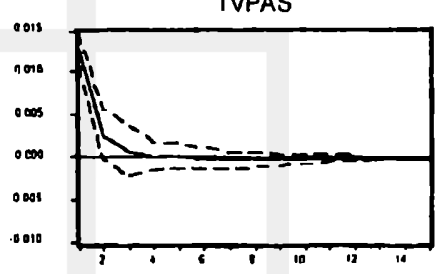

IPCN

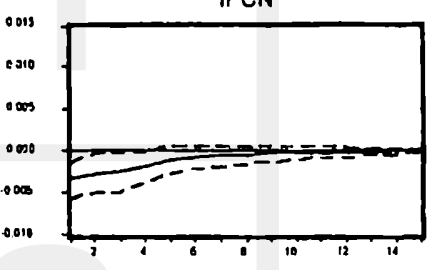

IVAE

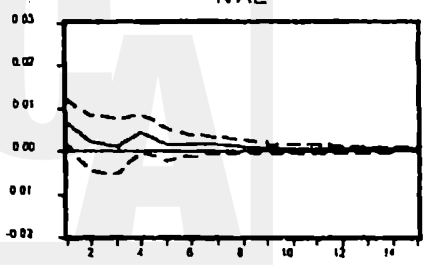

Aunque la tasa de interés no responde en forma contemporánea a una innovación positiva de los precios, éstos ejercen una fuerte y prolongada influencia sobre la primera, lo cual corresponde a la lógica económica. La tasa de interés es el precio del dinero y está muy relacionada con la evolución general de precios; en la medida en que estos suben, se reduce la oferta monetaria real, y con ello se incrementa la tasa de interés. 
Esta interpretación es consistente con el comportamiento del resto de variables en el caso de innovaciones positivas de IPCN.

En el segundo mes, ante la innovación inicial de IPCN, M3 responde en forma positiva, seguido como siempre de TVPAS. De ahi que la respuesta positiva de INAC no sea muy pronunciada en el segundo mes.

Pero M3 y TVPAS responden negativamenle en el tercer mes, lo cual supone una contradicción de la oferta monetaria real, que explica el repunte de INAC en dicho periodo.

El efecto de la restricción de la oferta monetaria real es un IVAE negativo durante los primeros tres meses.

\subsubsection{IVAE sobre INAC:}

La función de impulso respuesta que analiza los cambios en la tasa de interés producidos por una variación positiva de la actividad económica (ver Gráfica $\mathrm{N}^{0}$ 5), supone un impacto negativo no contemporáneo en la tasa de interés, alcanzando su mayor incidencia en el segundo mes, para volver casi en forma inmediata a la estabilidad. Su efeclo acumulado es negativo. La causalidad se muestra claramente en el análisis Granger, en donde se presenta una clara retroalimentación entre ambas variables (ver Ancxol).

Este resultado no entra en contradicción con la teoría económica, debido a que la tasa de interés y el producto nacional están relacionados en forma inversa (modelo IS-LM); por tanto, no resulta extraño que la suma de los multiplicadores de la respuesta de INAC ante innovaciones positivas de IVAE sea negativa. Aunque convencionalmente se acepla que la expansión de la actividad económica, implica un crecimiento en la demanda de saldos reales, que contribuye al incremento en la tasa de interés.
Griflea No. 4

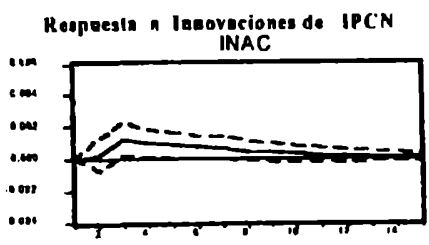

M3
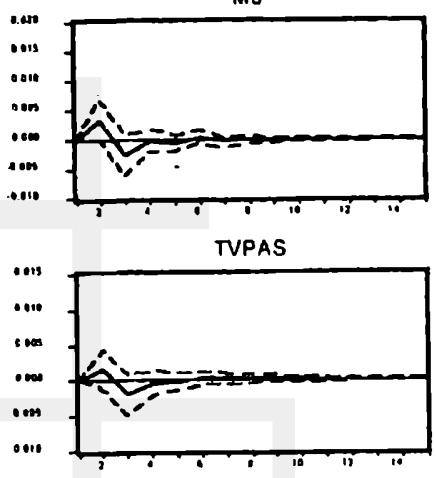

IPCN

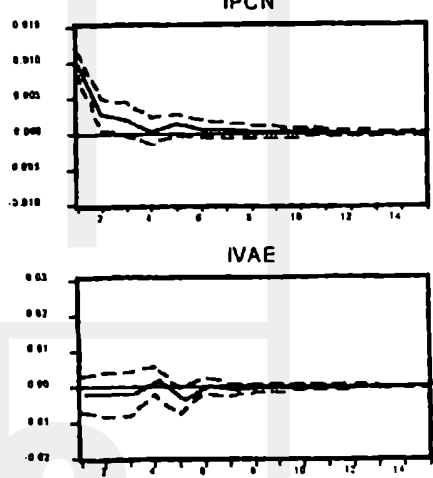

En cuanto a la respuesta de las otras variables ante una innovación positiva de IVAE, en las gráficas no se muestran con claridad las que corresponden a las variables monetarias, debido a que IVAE ocupa el último lugar en el ordenamiento y su impacto sobre las demás es menor; por tal razón, ninguna variable ofrece una respuesta contemporánea a dicha innovación. Contrariamente, el IVAE (revestido de una fuerte inercia), tiene una elevada respuesta positiva en el primer mes.

Sin embargo en el segundo mes, todas las variables se vuelven negativas, excepto los precios; M3 se reduce y es seguido de TVPAS. Esta combinación que permitirá cierta expansión, o al menos una menor reducción de la oferta monetaria real, se anula con el incremenlo de IPCN que acompaña a la caída de la esterilización. 
Esta contracción monetaria es acorde con la caída abrupta de IVAE en ese período y todo hace pensar en un incremento de la tasa de interés. Sin embargo esta última variable se vuelve negativa en el segundo mes, lo cual puede estar explicado en parte por la inercia de IVAE, que en ese período registra una caída más que notable, que justificaría la reducción de INAC.

Ya en el tercer mes se percibe una expansión de la oferta monetaria real (suben en la misma proporción M3 y el IPCN, y desciende fuertemente TVPAS), lo cual puede ser significativo para explicar el incremento de IVAE en ese periodo, aunque siempre hay que tomar en consideración la propia inercia de esta variable. Por otra parte, INAC se incrementa manifestando de nuevo un comportamiento contrario al esperado. En los meses siguientes prácticamente se estabilizan las variables.

Como no queda claro este proceso de ajuste, ya que INAC se comporta en forma contraria a lo esperado, se procedió a adelantar el IVAE en el ordenamiento, obteniéndose en este caso respuestas contemporáneas de M3 y TVPAS ante innovaciones positivas de IVAE, negativa en el primer caso y positiva en el segundo. Tal secuencia origina una reducción también contemporánea en los precios a nivel contemporáneo, que tiene como consecuencia una reducción en la oferta monetaria real en el primer mes, que sin embargo (al igual que el ordenamiento anterior) tampoco tiene repercusión sobre la tasa de interés.

De acuerdo a este último ordenamiento, en el segundo mes se estaria produciendo una expansión monetaria (suben M3 y los precios, y disminuye la esterilización) lo cual supone una expansión de la oferta monetaria real y con ella, la reducción en las tasas de interés que se registra en ese periodo, aunque IVAE mantiene la misma inercia que en el primer ordenamiento. En el tercer mes se estabilizan las variables.

Ante la falta de sintonia entre IVAE y M3 (M3 disminuye ante una subida de IVAE y viceversa), la disminución de la esterilización que de acuerdo al modelo, evoluciona de la misma forma que $\mathrm{M} 3$, se muestra ineficaz para expandir la oferta monetaria real, porque el IPCN se incrementa. Como se ha visto antes, la estèrilización es determinante de los precios, por lo que el IPCN crece ante la ausencia de TVPAS.

\subsubsection{Innovaciones de INAC}

En cuanto a la respuesta en las diferentes variables endógenas ante innovacioncs de INAC (vcr Gráfica № 6) sc presentan las siguientes IRFs:

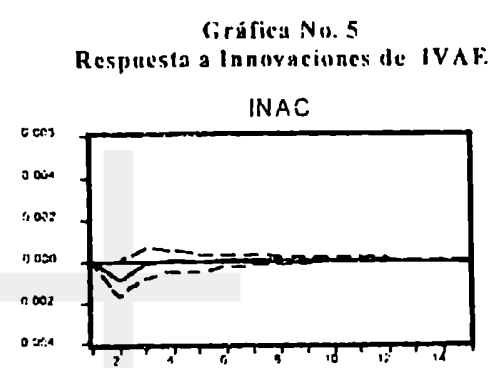

M3

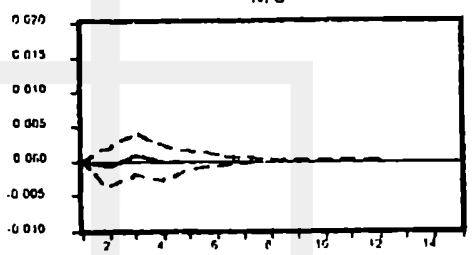

TVPAS

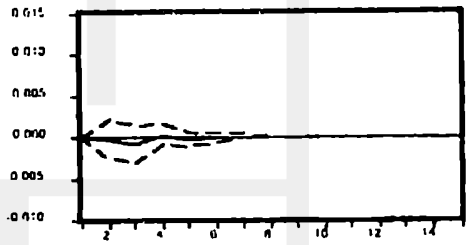

IPCN

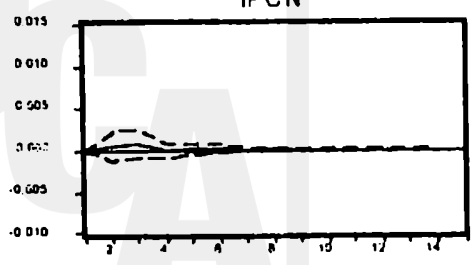

IVAE

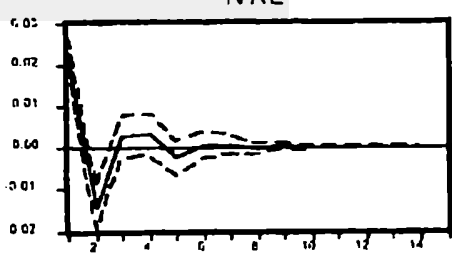


M3: En el caso de M3 se aprecia un ligero impacto positivo contemporáneo, que continúa en el segundo mes y que puede ser producto de la tendencia natural del capital ante una elevación de las tasas de interés; posteriormente M3 entra en un período de subidas y bajadas entre el tercer y el séptimo mes antes de estabilizarse, que podrían considerarse como "sobrereacciones" de M3, en parte atribuibles a su propia inercia, ante cambios en INAC. No obstante el impacto acumulado es negativo, tal como corresponde a lo establecido por la teoría económica, en donde un incremento de la tasa de interés deberá equilibrarse con una reducción de la oferta monetaria real, ya sea a través de la reducción de la oferta monetaria ó el incremento en los precios.

Esta relación no se refleja con claridad en las pruebas Granger, ya que las probabilidades de aceptar $\mathrm{H}_{0}$ son altas (ver Anexo 1).

TVPAS: La respuesta de TVPAS ante innovaciones en la tasa de interés, es positiva y contemporánea, y sigue la evolución de las respuestas de M3 ante cambios en INAC. La respuesta positiva de TVPAS ante INAC, estaría dentro de la lógica económica, en donde un incremento de la tasa de interés deberá acompañarse con una reducción en la cantidad de dinero, a fin de mantener el nuevo equilibrio sobre la curva de la demanda de saldos reales. Su efecto acumulado es positivo.

Cabe señalar que TVPAS reacciona en la misma forma y al mismo tiempo que M3. De acuerdo a este planteamiento, el modelo muestra que ante innovaciones positivas de INAC, cualquier incremento $\mathrm{M} 3$ es neutralizado por la política de esterilización.

Estas relaciones se confirman en la prueba Granger (ver Anexo 1). Existe una fuerte retroalimentación entre TVPAS e INAC.

IPCN: En el caso de la respuesta de IPCN ante innovaciones positivas en la tasa de interés, se había comentado al Iratar del Impulso Acumulado, que esta respuesta carecía de lógica económica, ya que ofrecía un valor acumulado negativo de IPCN ante cambios positivos de INAC. Este impacto negativo se presenta por el peso que tiene la respuesta de IPCN en los dos primeros meses.

Para interpretar esta disminución de IPCN, no hay que pasar por alto el comportamiento de las otras variables monetarias (M3 y TVPAS) ante innovaciones positivas en INAC, las cuales presentan respuestas positivas contemporáneas que continúan creciendo en el segundo mes, 'siendo siempre la respuesta de TVPAS superior a la de M3. La potencia de TVPAS provoca una reducción de los precios tanto en forma contcmporánea como en el segundo mes, demostrándose de nuevo la efcclivi-

Cirdfica Nill. h

Kespuesia de INAC: o Innoraciones de:

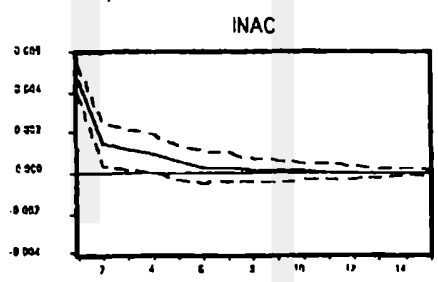

M3

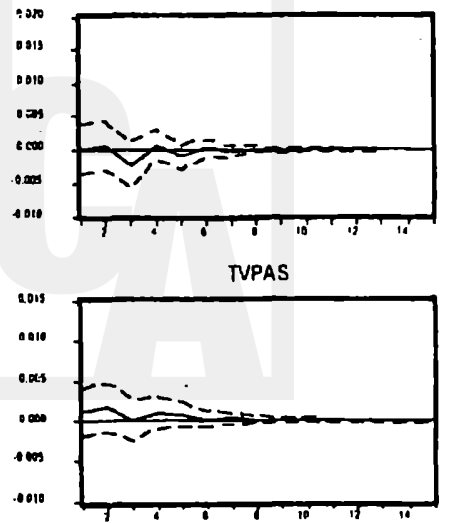
dad de la política de esterilización para el control de IPCN, que se reduce ó aumenta respectivamente. 
1).

La causalidad Granger no refleja esta rclación (ver Anexo

IVAE: Por último, las innovaciones en la tasa de interés provocan respuestas importantes en IVAE. Exislen signos evidentes de sobre reacciones de la actividad económica anle incrementos en la tasa de interés en parte explicados por la propia inercia del IVAE, con un efecto acumulado negativo, tal como corresponde a la leoría económica.

Sin embargo, la respuesta inicial positiva de IVAE ante un incremento de INAC (sin lógica cconómica) y su posicrior caida en cl segundo mes, no quedan totalmente claras, si bicn es cierto que TVPAS presiona a la baja a IPCN y ćslc supone una mejoría para IVAE.
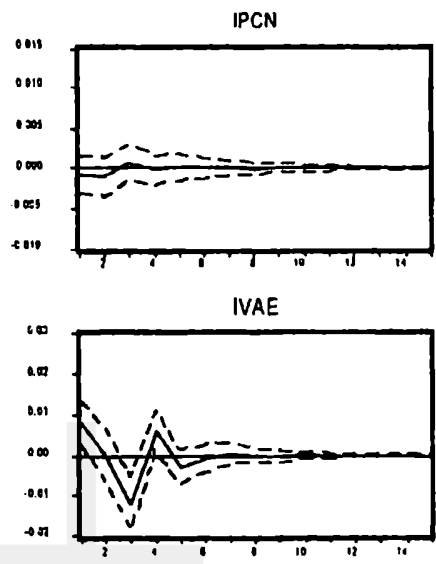

Ya en el tercer mes IVAE continúa su caída, pero ahí ya existe una clara contracción monetaria real (cae M3 y sube IPCN). En el $4^{0}$ mes, un incremento de $\mathrm{M} 3$, con el correspondiente crecimiento de TVPAS, contribuye al descenso de IPCN, y, por consiguiente, a una respuesta positiva de IVAE.

En general, las innovaciones positivas del IPCN, producen una reducción contemporánea y sostenida del IVAE, mientras que una innovación del IVAE, produce una leve subida del IPCN.

La causalidad Granger se presenta claramente en esta relación (ver Anexo 1).

\section{Conclusiones}

A lo largo del presente trabajo se ha tratado de describir las relaciones existentes entre las distintas variables endógenas del modelo. Como se ha podido apreciar, sobre la base de los resultados obtenidos en las IRFs se ha tratado de pormenorizar la dinámica de funcionamiento del sistema. Aunque algunos de los planteamientos manejados en los puntos anteriores, requerirían de estudios más profundos sobre el particular. No obstante, se pueden presentar algunas conclusiones provisionales, en torno a los aspectos más relevantes que centralizan el debate actual.

\subsection{Sobre el funcionamiento del sistema.}

El crecimiento de las tasas de interés tiene un impacto bastante perjudicial en la economía salvadoreña. De acuerdo con el modelo aplicado, ofrece unos resultados de exceso ude esterilización, estancamiento económico y deflación. Esta última producida por el impacto de la política de esterilización (Gráfica No. 6). Esta interpretación prácticamente viene a coincidir con los resultados de otros estudios sobre la materia.

Por otra parte, la tasa de interés no está determinada aparentemente por las OMAS como se sostiene en muchas ocasiones, sino por la contracción o expansión de la oferta monetaria real, que es producto de la combinación específica de 
inflación, oferta monetaria y esterilización, que se presenta en cada unas de las funciones de impulso respuesta; incrementándose la tasa cuando existe contracción monetaria y reduciéndose cuando se producen expansiones. Por su parte, la actividad económica en general, presenta respuestas positivas en los meses en que se produce una expansión de la oferta monetaria real, y negativas en aquellos períodos donde se da una contracción (Gráficas Nos. 2,3,4). Todos estos razonamientos están dentro de la lógica económica.

Lo mismo ocurre en cuanto a los precios y la actividad económica. De acuerdo con el modelo, una reducción de IPCN es acompañada en buena medida por un crecimiento en IVAE; mientras que una innovación positiva en esta última variable, incrementa IPCN (Gráficas Nos. 2,3,4 y 5).

Aquí se plantea un punto interesante, ya que en El Salvador en los últimos años, varios estudios ponen en contraposición la deflación existente con la contracción de la actividad económica, llegándose en muchos casos a proponer el intercambio de crecimiento por precios, es decir, un poco menos de estabilidad a cambio de más desarrollo.

Sin embargo, de acuerdo a nuestra interpretación del modelo, el crecimiento del producto incrementa ligeramente los precios y aparentemente no se le presentarían muchos problemas a la economía nacional para absorber tales incrementos. Pero la dirección de la causalidad Granger se presenta claramente de la inflación sobre la actividad económica y no al revés, e implica de acuerdo a la IRF, que el incremento de precios supondrá reducciones en el producto. Por tanto, un aumento de precios no tendría impacto positivo en el crecimiento, pero éste último conlleva incrementos en los _precios, que si se manipulan pueden originar distorsiones en el sistema.

\subsection{Sobre la incidencia de la esterilización en la tasa de interés.}

De acuerdo con los resultados obtenidos en el modelo, a largo plazo, la respuesta de INAC ante variaciones positivas del resto las variables endógenas, responde a los principios de la teoría económica, excepto en 2 casos:

\subsubsection{El incremento de la oferta monetaria nominal presiona a la tasa de interés al alza.}

Esto se explica porque el incremento de la masa monetaria es contrarrestado con la política de esterilización de las autoridades, cuyo resultado conjunto supone una contracción monetaria real, con su correspondiente alza en la tasa de interés y la disminución de la actividad económica (Gráfica $\mathrm{N}^{2} 2$ ).

\subsubsection{El incremento de la esterilización presiona a la reducción de la tasa de interés.}

La razón de ello es que el incremento de la esterilización provoca un fuerte impacto negativo en los precios, cuyo resultado neto es una expansión monetaria real, con la correspondiente disminución de intereses y alzas en la actividad económica (Gráfica № 3). 
El incremento de TVPAS, presente en ambas situaciones, causa distintos efectos debido a que el incremento de M3 no causa respuesta contemporánea importante en IPCN (sino hasta el tercer mes), mientras que una innovación positiva en TVPAS ejerce un prolongado efecto negativo sobre el IPCN desde el primer mes.

\subsection{Sobre la incidencia de la tasa de interés en la actividad económica.}

La tasa de interés parece ser reflejo del mercado monetario (oferta monetaria nominal, esterilización y precios) y en buena medida resulta significativa para explicar la respuesta de la actividad económica; se considera que, en general, un crecimiento en las tasas de interés está asociado a reducciones en IVAE, y un decrecimiento en INAC a expansiones de la actividad económica (Gráficas Nos. 2,3,4). Esta interpretación es coincidente con la teoría económica.

\subsection{Sobre la relación entre el mercado real y el mercado monetario.}

$\mathrm{El}$ análisis que más dificultades ha presentado, es el de las respuestas que causa la actividad económica sobre las variables monetarias, en el cual se revisaron incluso varios ordenamientos.

El resultado ha sido la constatación de las dificultades para adecuar ambos mercados, el real y el monetario. Parte de estas dificultades, radica en las oscilaciones propias de la inercia de IVAE y de M3 en cada período. Sin embargo, se presentan elementos comunes en todos los ordenamientos, que se resumen en su Impulso Acumulado, según el cual, ante innovaciones positivas de la actividad económica, disminuye la oferta monetaria nominal y la esterilización, y suben los precios. Esta situación se refleja en el primer o segundo mes en función del ordenamiento adoptado.

En cuanto a IVAE, que adolece de fuertes oscilaciones producto de su inercia, cae fuertemente en el segundo mes y luego se estabiliza; INAC por su parte sigue una evolución similar. (Gráfica № 5).

\subsection{Sobre la incidencia de las variables exógenas.}

En cuanto a las variables exógenas, aplicando el análisis Granger se puede deducir lo siguiente:

a) Encaje Legal: Presenta relaciones claras de causalidad Granger con la tasa de interés (ver Anexo 1), por lo que se revela que la política monetaria de largo plazo (el encaje legal) es eficaz para determinar la tasa de interés. Así mismo al igual que OMAS, muestra que los cambios en el encaje son registrados por los precios.

b) Tasa de Interés e Inflación Internacionales: En teoría deberían tener un fuerte impacto en las variables homólogas nacionales, sin embargo, el análisis Granger no muestra relaciones de causalidad claras con INAC e IPCN. Sin embargo, las variaciones en BONOS e IPCUSA sí son registradas por IVAE y TVPAS (ver Anexo 1). 
Anexo 1

\section{Resultados Test causalidad de Granger (Período 1991: 04 1999: 03)}

\begin{tabular}{|c|c|c|c|c|c|}
\hline \multicolumn{6}{|c|}{ REZAGOS } \\
\hline ULA & $\frac{1}{\text { Probabilidad }}$ & $\begin{array}{c}3 \\
\text { Probabilidad }\end{array}$ & $\begin{array}{c}6 \\
\text { Probabilidad }\end{array}$ & $\begin{array}{c}9 \\
\text { Probabilidad }\end{array}$ & $\begin{array}{c}12 \\
\text { Probabilidad }\end{array}$ \\
\hline $\begin{array}{l}\mathrm{N} \\
\mathrm{C}\end{array}$ & & & & & $\begin{array}{c}0.247220507 \\
0.44326654 \\
\end{array}$ \\
\hline & & & & & 189 \\
\hline$c$ & 0943588 & $\begin{array}{l}0.570981979 \\
0.270851928 \\
\end{array}$ & $\begin{array}{l}0.161255525 \\
0.100124423 \\
\end{array}$ & $\begin{array}{c}0.60855939 \\
0.366483615\end{array}$ & $\begin{array}{l}0.599561553 \\
0.589002804\end{array}$ \\
\hline C & $\begin{array}{l}879 \\
158 \\
\end{array}$ & $\begin{array}{l}0.693503106 \\
0.002243247 \\
\end{array}$ & $\begin{array}{l}474 \\
006\end{array}$ & $\begin{array}{l}02 \\
23 \\
\end{array}$ & $\begin{array}{l}0.229336447 \\
\mathbf{0 . 0 8 2 1 8 4 5 7 3} \\
\end{array}$ \\
\hline AC & 0.223441749 & 44758699 & .685242959 & 346887028 & 0.020482012 \\
\hline $\mathrm{AC}$ & 6 & 4 & 7 & 36 & 39 \\
\hline re INAC & 0.045020277 & 53824453 & 5447 & 86290157 & .28716035 \\
\hline $\begin{array}{l}\mathrm{E} \\
\mathrm{N}\end{array}$ & $\begin{array}{l}\mathbf{0 . 0 8 6 8 7 0 6 3 3} \\
0.777292069\end{array}$ & & & & $\begin{array}{l}7144 \\
4804\end{array}$ \\
\hline (1) & $\begin{array}{r}0.383611748 \\
0.79961866 \\
\end{array}$ & & $\begin{array}{l}0.105864248 \\
0.064720756 \\
\end{array}$ & & $\begin{array}{l}186679944 \\
568146941 \\
\end{array}$ \\
\hline $\begin{array}{l}\mathrm{CN} \\
\text { VPA } \\
\end{array}$ & $\begin{array}{r}97 \\
35 \\
\end{array}$ & & & $\begin{array}{l}391 \\
735 \\
\end{array}$ & $\begin{array}{l}0.023186825 \\
0.037259255 \\
\end{array}$ \\
\hline N & 6 & 75 & 88 & 8 & $(12$ \\
\hline CUS & 0.10515 & 475 & 182 & 177 & 600583538 \\
\hline NCAJE no cal & 0.638678462 & 9559 & 7394 & 17607552 & 0.181520068 \\
\hline 13 no causa Granger sobre IVAE & & & & & \\
\hline VPAS no & $\begin{array}{c}0.8432105856 \\
0.84621876 \\
\end{array}$ & & & & $\begin{array}{l}0.36328108 .5 \\
0.698818073 \\
\end{array}$ \\
\hline 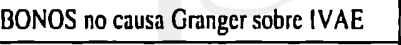 & 4 & 13 & & 24 & 77 \\
\hline CUSA causa Granger sobre IVAE & 0.021314019 & 0.016484478 & 0.113324294 & 0.176717433 & 0.322896255 \\
\hline VCAJE no causa Granger sobre IVAE & 0.668118314 & 0.793162469 & 0.708 & 0.884620313 & 0.836079888 \\
\hline VPA & & & & $\begin{array}{l}568 \\
723 \\
\end{array}$ & $\begin{array}{l}0.71110987162 \\
0.076798526 \\
\end{array}$ \\
\hline 13 & 641 & 52 & 0.282516423 & 0.059039107 & $.25537(1) 14$ \\
\hline CUSA no causa Granger sobre MB & 489 & 1798 & 0.696782877 & 0.49521264 & 0.083856663 \\
\hline NCAJE no causa Granger & 0.283508322 & 0.2617119613 & $0.52(149965$ & 0.3367509016 & 0.499975977 \\
\hline ONOS no causa Granger sobre TVPAS & 0.448 & 0.72 & 0.575 & $\overline{495}$ & 0.124 \\
\hline CUSA no causa Granger sobre TVPAS & 0.793114746 & 0.030494414 & 0.032104349 & 0.2117537722 & 010517607 \\
\hline NCAJE causa Grangcr sobrc TVPAS & .396186421 & 0.4345931163 & 0.841772948 & .158757602 & 0.103060859 \\
\hline CUSA no & $\begin{array}{r}0.7073669666 \\
0.68(09) 344\end{array}$ & & & $\begin{array}{l}0.148492836 \\
0.311490897\end{array}$ & $\begin{array}{l}(0.34248 .3101 \\
(0.745528 .367\end{array}$ \\
\hline
\end{tabular}




\section{Referencias bibliográficas}

Acevedo, Carlos. 1998. "Actores, Agendas y Escenarios en El Salvador 1998"

Dornbush, Rudiger y Fischer, Stanley. 1994. "Macroeconomía" Editorial McGraw Hill (6a. Edición en español).

Fernández, Andrés et al. 1995. "Política Económica"Editorial McGraw Hill (1ª. Edición ).

Gujarati, Damodar N. 1995. "Econometría"Editorial McGraw Hill (3². Edición en español)

Krugman, Paul y Obstfeld, Maurice. 1995. "Economía Internacional. Teoría y Política" Editorial McGraw Hill (3". Edición en español)

Maddala, G.S. 1996. "Introducción a la Econometría" Editorial Prentice Hall ( $1^{\text {a }}$. Edición en español)

Mascareñas, Juan et al. 1996. "Mercado de Divisas y Análisis de Mercado Financiero". Editorial Pirámide (1". Edición).

Sachs, Jeffre y D. Y Larraín, Felipe. 1994 "Macroeconomía en la Economía Global" Editorial Prentice Hall (1". Edición en español).

Andersson, Paul A. "Help for the Regional Economic Forecaster: Vector Autoregression". Work Paper, Research Department, Federal Reserve Bank of Minneapolis.

Demirgüq-Kunt, Asli y Huizinga, Harry. 1997. "Determinants of Commercial Bank Interest Margins and Profitability: some International Evidence". Development Research Group. The Word Bank.

Hurtado, Xiomara y Osorio, Juan A. 1998. "Determinantes de la Inflación en el Salvador" Boletín Económico \#122. Banco Central de Reserva.

Tood, Richard M. "Improving Economic Forecasting with Bayesian Vector Autoregression" Work Paper, Research Department, Federal Reserve Bank of Minneapolis.

Tood, Richard M. "Vector Autoregression Evidence on Monetarism: Another look at the Robustness Debate".

Work Paper, Research Department, Federal Reserve Bank of Minneapolis and Associate Director Institute for Empirical Macroeconomics.

FMI. "El control del crédito y la liquidez externos"

Página Web del Banco Central de Reserva de El Salvador (http://www.ber.gob.sv.f)

Página Web del Federal Reserve Board of Governors.

Página Web del U.S. Department of Labor. Bureau of Labor Statistics. Washington, D.C. 20212.

Revistras Trimestrales, Banco Central de Reserva, 1990-1998

Archivo de Ayuda Técnica (Help) del programa Econometric Views v.2.0)

\section{Notas}

1. De acuerdo al principio de Paridad del Poder Adquisitivo (PPA) y la Condición de Paridad de Intereses (CPI), en economías ahiertas y libre movilidad de capitales, 
con tipo de cambio fijo, el diferencial entre tasas de interés e inflación nacionales no puede alejarse demasiado del existente a nivel internacional, sin riesgo de incurir en graves desequilibrios:

$$
\mathbf{i}-\boldsymbol{\pi}=\mathbf{i}^{*}-\pi^{*}
$$

2. El Ministro de Hacienda de la pasada administración, ha venido sosteniendo que la no dolarización atenta contra la reducción de las tasas de interés.

3. Al igual que se exige a otros sectores económicos, la banca también deberia mostrar niveles de competitividad internacional, ya sea reduciendo sus costos, lo que implicaría disminución en sus márgenes de intermediación y por tanto en las tasas activas, ó bien, mejorando la calidad de sus servicios.

4. Serie proporcionada por el Departamento de Operaciones Monetarias del BCR

5. Indice estimado por la Dirección General de Estadística y Censos del Ministerio de Economía

6. Serie proporcionada por el Departamento de Cuentas Macroeconómicas del BCR

7. Serie proporcionada por el Departamento de Cuentas Macrocconómicas del BCR

8. La información de esta variablc ha sido cedida para el presente estudio por Xiomara Hurtado de García y Juan Antonio Osorio Mcjía, del Departamento de Desarrollo Financiero del BCR.

9. Edición H.15 - página Web del Federal Rescrve Board of Governors

10. La información de esta serie fuc extraída de la página Web del U.S. Departament Of Labor, Bureau of Labor Statistics, Washington, D.C.

11. La información base para la claboración de esta scric ha sido proporcionada por cl Departamento de Opcraciones Monetarias del BCR y la revista trimestral de dicha institución.

12. El criterio de Akaike AIC (Akaike Information Criteria, por sus siglas en inglés), cs una guía para la selección del número de rezagos a utilizar en la ccuación; que penaliza por la incorporación de cocficientes extras en el modelo. Sc basa en la suma de los cuadrados de los residuos. Bajo ciertas circunstancias, permitc cscoger el número óptimo de rezagos, cligicndo cntrc dos modelos, aquel que presentc el menor valor de AIC.

13. Es un criterio alternativo al AIC, utilizando básicamente la misma interpretación pero con mayor penalización por la incorporación de coeficientes extras en cl modelo.

14. Prucba del tipo máxima vcrosimilitud (Log Likclihood). Estc critcrio es el cocicntc de máxima verosimilitud que sc basa en la tcoría asintótica. La prucba utiliza la matriz de varianzas y covarianzas de las innovaciones de VAR cvaluado con los rezagos que se desec comparar.

15. Variante de la prucba LR propucsta por Enders

16. El ordenamicnto de las variables en el VAR no condiciona cl valor estimado para los coeficientes, en cambio el ordenamiento en las IRFs si hace variar las respucstas de las variables endógenas ante innovaciones en una de cllas.

17. El sentido de causalidad Granger no se debe interpretar como una razón de causa Æ efecto entre dos scries de ticmpo; al afirmarse que una variable A causa Granger en otra variable B, se debe entender que la primer variable influye en la detcrminación del valor de la scgunda. Sin embargo, csto no neccsariamente implica que al cxistir causalidad entre dos variables, equivale a decir que una de cllas cs el cfecto de la otra. La econometría es rica en tipos de relaciones entre variables, pero la 
mayoría de ellas son de tipo espúreo, es decir, que no tienen ninguna validez o fundamento.

La prueba Granger se basa en realizar varias regresiones entre las variables con distintos términos en rezago, y de acuerdo a los resultados obtenidos se establecen si existe la presencia de Causalidad Unidireccional (en cualquiera de las dos direcciones), retroalimentación o causalidad bilateral (existe causalidad Granger en ambas direcciones) ó independencia entre las dos series de tiempo (no existe causalidad Granger en ambas direcciones) ó independencia entre las dos series de tiempo (no existe causalidad Granger en ambas variables). Los resultados de las pruebas Granger pueden observarse en el ANEXO 1, aplicados de 1 a 12 rezagos. En la medida en que se mantenga la probabilidad de rechazo de la hipótesis nula para los diferentes rezados, así como la tendencia de dicha probabilidad, se estará confirmando la consistencia de las relaciones de causalidad entre las variables.

18. Xiomara Hurtado y Juan Antonio Osorio. "Determinantes de la inflación en El Salvador", Bolctín Económico \#122, septicmbre 1998.BCR. 